\title{
O papel dos Pontos de Troca de Tráfego em políticas e regulação da banda larga
}

The Role of Internet Exchange Points in Broadband Policy and Regulation

Submetido(submitted): 23 de agosto de 2010

Parecer(revised): 18 de setembro de 2010

Aceito(accepted): 12 de outubro de 2010

Daniel Brandão Cavalcanti*

\section{Resumo}

Novos investimentos em infraestrutura de redes de transporte (backbones) para a internet e a necessidade de trocar tráfego com backbones existentes, tanto na modalidade de trânsito como na de peering, indicam a crescente relevância de um elemento-chave nessa arquitetura - o Ponto de Troca de Tráfego (PTT). Há muito poucos PTT disponíveis hoje em dia, principalmente se considerado o vertiginoso crescimento no número de sistemas autônomos anunciados. Os PTT implantados por redes de pesquisa demonstram que um acréscimo expressivo no número desses pontos teria o efeito de melhorar a topologia da internet, aumentar a conectividade, reduzir a latência e o custo de troca de tráfego. Adicionalmente, os PTT podem ser o local mais eficiente para a oferta de determinados serviços. Este trabalho aborda as possibilidades de ação regulatória para estimular a implementação dos PTT e para assegurar que seus benefícios sejam amplamente compartilhados.

\section{Abstract}

New investments in Internet backbone infrastructure and the need to exchange traffic with existing backbones, on a peering or transit basis, point to the emergence of a key infrastructure element - the Internet Exchange Point (IXP). There are very few IXPs available today, considering the huge increase in the number of autonomous systems advertised. IXPs deployed by research networks demonstrate that a substantial increase in their number would improve the Internet topology, increase connectivity, reduce latency and the cost of traffic exchange. Additionally, IXPs can be efficient locations from which to offer system services. This paper discusses possibilities of regulatory action to stimulate the implementation of IXPs and to ensure that their benefits are widely shared.

Palavras-chave: política pública; regulação; banda larga; internet; ponto de troca de tráfego. Keywords: policy; regulation; broadband; Internet; Internet exchange point.

*M.Sc. em Engenharia Elétrica pela University of Waterloo, Canadá. Especialista em Regulação de Telecomunicações e Engenheiro Eletricista pela Universidade de Brasília (UnB). Gestor Governamental, atualmente na Secretaria de Telecomunicações do Ministério das Comunicações.

Revista de Direito, Estado e Telecomunicações, v. 3, n. 1, p. 75-88 (2011) 


\section{Introdução}

Ao longo dos dois últimos anos, diversos países divulgaram planos nacionais de banda larga, alguns dos quais propõem a implantação de extensas redes de transporte (backbones) de fibra ótica como forma de assegurar cobertura a áreas não atendidas ou mal atendidas. Em alguns países, esse novo backbone é visto como elemento essencial para estimular a competição no mercado de atacado de redes de transporte IP para tráfego na internet. Entretanto, essas redes precisam ser capazes de trocar tráfego com outros backbones, por meio de peering, assim como fornecer pontos convenientes onde Provedores de Serviço de Internet (PSI) e operadoras de rede possam se interconectar para a compra e venda de capacidade de trânsito. Todas essas condições indicam a crescente relevância de um elemento-chave de infraestrutura, que é o Ponto de Troca de Tráfego (PTT).

\section{O conceito de PTT}

Um PTT é um local com instalações físicas em que provedores de serviços e operadoras de rede podem trocar tráfego entre suas redes, que são identificadas como Sistemas Autônomos [Autonomous Systems (AS)]. Os primeiros PTT abertos e neutros do ponto de vista da possibilidade de neles participar e do local onde estão instalados foram implantados no início da década de 1990. Um PTT típico consiste em um ou mais switches e roteadores, aos quais cada um dos participantes se conecta. Ao trocar parte de seu tráfego diretamente, por meio de peering, as redes podem reduzir a proporção de seu tráfego total que precisa ser entregue aos provedores de capacidade de trânsito. Assim, é possível reduzir os custos de seu serviço e aumentar o desempenho conjunto das redes interconectadas.

As funcionalidades de trânsito e de peering diferenciam-se essencialmente pelo fato de que, em uma relação de trânsito, uma rede paga à outra pela interconexão e, portanto, torna-se uma cliente de atacado da outra rede. Diferentemente, em uma relação de peering, as redes que vendem capacidade de trânsito a terceiros irão realizar o roteamento do 
tráfego de seus clientes de trânsito entre si, por meio de trocas diretas e sem pagamento entre os parceiros de peering. ${ }^{1}$

A estrutura do PTT fornece os benefícios adicionais de melhoria na eficiência de roteamento, aumento da tolerância a falhas, redução de latência e menor demanda por capacidade de transporte de dados. O tráfego trocado diretamente via peering num PTT normalmente não é objeto de cobrança, diferentemente do tráfego de trânsito. A Figura 1 ilustra o papel desempenhado pelos PTT no provimento de acordos de peering, de forma a viabilizar a troca direta de tráfego entre os participantes, bem como capacidade de trânsito nos backbones presentes naquele ponto. ${ }^{2}$

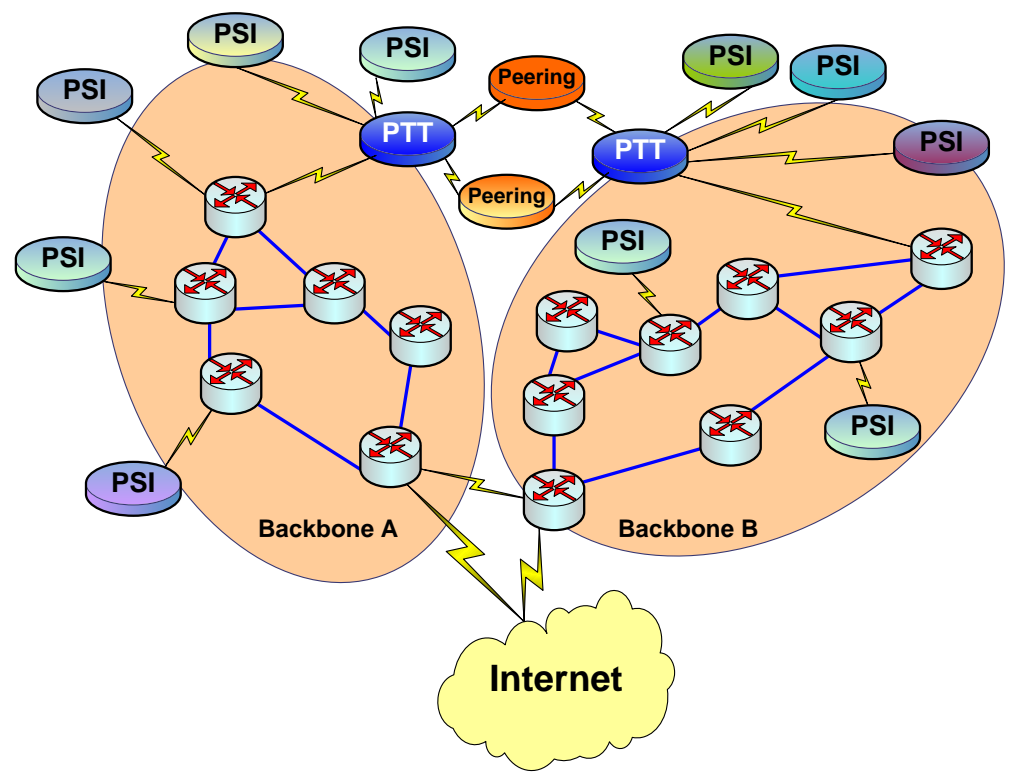

Figura 1 - $O$ conceito de Ponto de Troca de Tráfego (PTT)

${ }^{1}$ KENDE, M. (2000). The Digital Handshake: Connecting Internet Backbones. In: FCC OPP Working Papers 32.

${ }^{2}$ CISCO. The Keystone of Internet Economy, Next Generation Interconnection. In: Cisco Networkers (2001-2005). 
Como pode ser observado na Figura 1, a partir da existência do PTT, um Provedor de Serviço de Internet (PSI) independente não é mais cativo do backbone A ou do backbone $\mathrm{B}$ na compra de capacidade de trânsito. Ao se interconectar no PTT, o PSI passa a ter a opção de escoar seu tráfego para a internet por um ou outro backbone, e até mesmo por ambos. Paralelamente, o PSI tem a possibilidade de intercambiar parte de seu tráfego diretamente com os demais PSI, por meio de acordos de peering. Todos esses fatores combinados, e que são propiciados pelos PTT, claramente aumentam o poder de barganha dos PSI na contratação de capacidade de trânsito nos backbones.

Atualmente os PTT tendem a ser implementados como resultado do esforço de cooperação entre empresas, por comunidades acadêmicas e de pesquisa, ou por governos. No entanto, considerando a atual topologia da internet e o crescimento no número de sistemas autônomos anunciados que recentemente passaram a receber endereços de 32 bits, o que permite a existência de um número ainda maior de $A S$ - há muito poucos PTT disponíveis hoje em dia.

A implantação de PTT por redes de pesquisa tem demonstrado que um aumento significativo no número desses pontos de intercâmbio teria o efeito de melhoria na topologia da internet, aumentando a conectividade, reduzindo a latência e o custo de troca de tráfego. Adicionalmente, os PTT podem se constituir em locais eficientes para a prestação de serviços de gestão de redes. A Figura 2 ilustra como a topologia da internet tem evoluído nos últimos anos de uma estrutura hierárquica tradicional para uma estrutura bem mais complexa. 


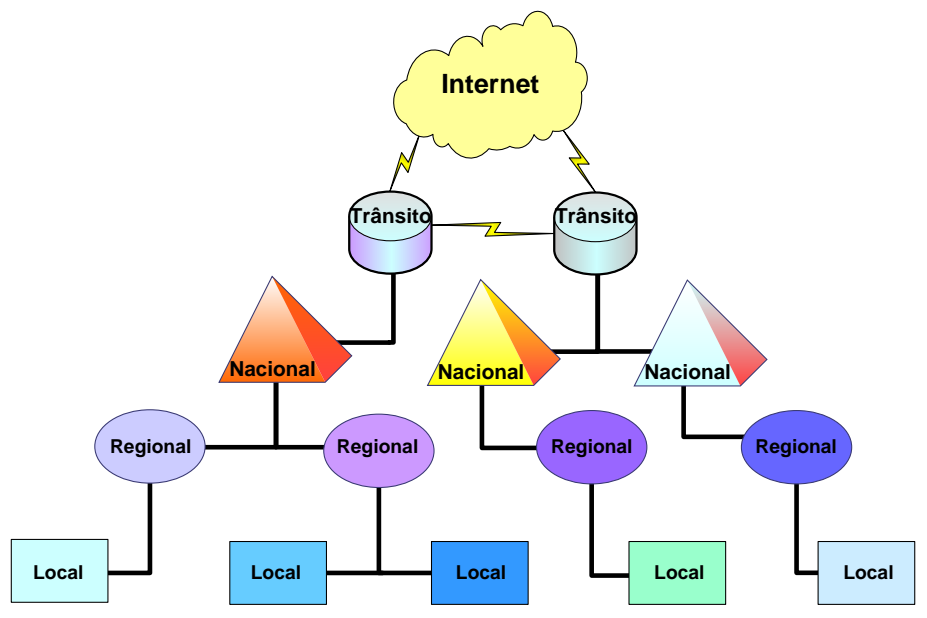

Figura 2 - Topologia da internet: evoluindo da estrutura hierárquica tradicional (acima) para uma estrutura complexa (abaixo)

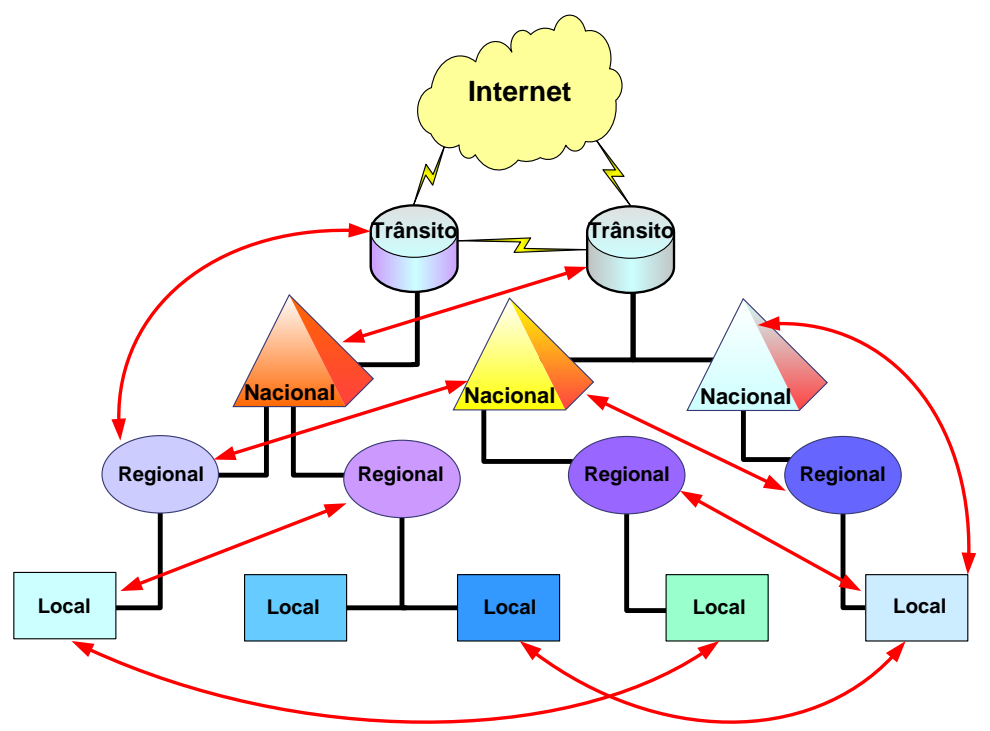

Revista de Direito, Estado e Telecomunicações, v. 3, n. 1, p. 75-88 (2011) DOI: https://doi.org/10.26512/lstr.v3i1.21666 
A Internet hoje, cada vez menos, corresponde à visão idealizada de uma estrutura hierárquica tradicional. Na prática, a Internet se apresenta como uma estrutura bastante mais complexa, onde a interação entre os diversos sistemas autônomos pode ser estabelecida diretamente em vários locais, tanto em trocas bilaterais como multilaterais, tipicamente nos PTT.

A utilidade de um PTT está diretamente relacionada ao número de redes que estão presentes naquele dado ponto de troca. No entanto, os grandes backbones nacionais tipicamente interconectam-se em um número muito reduzido de PTT. Dados os claros benefícios dos PTT, isso parece indicar que há espaço para ação regulatória visando estimular a implementação de PTT.

O Comitê Gestor da Internet no Brasil (CGI.br), que foi criado em 1995 numa iniciativa do Ministério das Comunicações e do Ministério da Ciência e Tecnologia, por meio da Portaria Interministerial n. 147, com alterações no Decreto Presidencial n. 4.826/2003, é constituído por membros de governo, do setor empresarial, do terceiro setor e da comunidade acadêmica. O CGI atualmente é responsável pela maior iniciativa de implantação de PTT no Brasil - denominada PTTMetro ${ }^{3}$ - com pontos de presença nas maiores cidades do país.

No início de 2010, o Brasil contava com 20 PTT abertos, localizados em 12 cidades. Somente a cidade de São Paulo abrigava oito desses PTT. O número de participantes em cada PTT varia enormemente, desde um mínimo de 4 participantes no menor PTT, até 94 no maior PTT do país. Para que se possa estabelecer um parâmetro de comparação, de acordo com a European Internet Exchange Association ${ }^{4}$, em outubro de 2009, a Europa dispunha de 389 PTT distribuídos em 115 cidades de 33 países.

\section{Tendências atuais}

O uso dos PTT como parte de uma estratégia para simultaneamente estimular o aumento na penetração da banda larga, reduzir custos e prover

${ }^{3}$ GETSCHKO, D.; MOREIRAS, A. (2008). Os Pontos de Troca de Tráfego, o PTT Metro e a internet brasileira. In: poliTICS 1: 15-21.

${ }^{4}$ EURO-IX. (2009). Report on European IXPs - 2009. European Internet Exchange Association.

Revista de Direito, Estado e Telecomunicações, v. 3, n. 1, p. 75-88 (2011)

DOI: https://doi.org/10.26512/lstr.v3i1.21666 
condições mais equitativas para a realização de interconexão IP tem sido o foco de diversos projetos nos últimos anos. Uma das primeiras iniciativas no gênero foi o Projeto Atlas [Accessing Telecoms Links Across Scotland], que foi implementado em 2003, tendo como objetivos melhorar o acesso da Escócia à internet global e reduzir os altos custos das redes de transporte de dados em banda larga. ${ }^{5}$ Esse projeto extremamente inovador estabeleceu os PTT [ou Telecoms Trading Exchange (TTE)] com pontos de presença localizados em três cidades - Glasgow, Edinburgo e Aberdeen - contendo switches operados por entidades neutras no processo de troca de tráfego. Adicionalmente, o projeto fornecia capacidade de backhaul até Londres, de forma que os PTT se constituíssem em locais onde se pudesse negociar, em bases competitivas e em um ambiente aberto a qualquer interessado, tanto capacidade de trânsito como conectividade em banda larga.

Diversos pesquisadores têm realizado trabalhos recentes abordando a questão da interconexão IP no contexto de redes de próxima geração [Next Generation Networks - NGN]. O European Regulators Group (ERG) ${ }^{6}$ aponta que, como resultado da migração para um ambiente NGN, as operadoras de telecomunicações dominantes [incumbents] estarão menos interessadas em estabelecer presença em pontos neutros de interconexão, e estarão mais propensas a preservar um sistema que mantém, e até amplia, a condição assimétrica em seu favor.

Como também indica outro estudo, encomendado pela Comissão Européia $^{7}$, com a migração para redes NGN, as incumbents tem uma oportunidade para reduzir seu número de pontos de interconexão IP. Embora se saiba que, nesse novo ambiente, tanto o acesso como a interconexão serão implementados utilizando IP, isso não significa que serão realizados necessariamente nos mesmos locais. Um conflito potencial surge pelo fato de que as incumbents que provêem capacidade de transporte IP em seus backbones não desejarão que as operadoras que provêem acesso IP na

${ }^{5}$ ATLAS. (2002). Connecting Scotland: our broadband future - Making it Happen. Scottish Executive.

${ }^{6}$ ERG. (2007). Project Team on IP-Interconnection and NGN: Final Report. European Regulators Group.

${ }^{7}$ MARCUS, J.; ELIXMANN, D. (2008). The Future of IP Interconnection: Technical, Economic, and Public Policy Aspects. WIK Consult, European Commission. 
"última milha" estejam visíveis no nível de roteamento IP. Em outras palavras, as incumbents não terão qualquer incentivo para prover pontos neutros para interconexão.

$\mathrm{Na}$ questão relativa a investimento em banda larga e regulação, um trabalho recente ${ }^{8}$ de revisão da bibliografia nesse tema aponta para um iminente conflito entre regulação, competição e investimento. As operadoras dominantes tendem a encarar a intervenção regulatória como sendo excessiva. No entanto, tanto no atacado como no varejo, medidas regulatórias podem criar condições favoráveis que induzam investimentos pelos demais atores de mercado.

No âmbito de estudos ${ }^{9}$ relacionados ao Plano Nacional de Banda Larga dos EUA, foi realizada pesquisa em diversos países acerca das políticas públicas de estímulo à internet banda larga. Constata-se que a regulação pode ser importante ferramenta na promoção da adoção de banda larga, e que mecanismos que assegurem facilidades na interconexão com incumbents podem aumentar os níveis de investimento e de oferta do serviço. Todas as operadoras de redes de telecomunicações têm obrigações de prover interconexão, e requisitos adicionais devem ser estabelecidos para operadoras com poder de mercado significativo (PMS) ou que controlem um recurso crítico, ou gargalo, do ponto de vista da competição. A regulação de preços deve estar incluída dentre tais requisitos. Outros remédios considerados essenciais para assegurar competição nesse mercado também devem ser implementados, tais como a "regra da simultaneidade" adotada na Alemanha, que veda a prestação de novos serviços por operadoras detentoras de PMS a menos que seus competidores tenham a oportunidade de prestar serviço similar.

${ }^{8}$ CAMBINI, C.; JIANG, Y. (2009). Broadband Investment and Regulation: A Literature Review. In: Telecommunications Policy 33: 559-574.

${ }^{9}$ BERKMAN CENTER FOR INTERNET \& SOCIETY. (2009). Next Generation Connectivity: A Review of Broadband Internet Transitions and Policy from Around the World. Harvard University.

Revista de Direito, Estado e Telecomunicações, v. 3, n. 1, p. 75-88 (2011)

DOI: https://doi.org/10.26512/lstr.v3i1.21666 


\section{Aspectos Regulatórios e Soluções}

Um estudo bastante recente ${ }^{10}$ nas áreas de regulação, de políticas públicas e de investimento em infraestrutura de comunicações chega à conclusão de que políticas eficazes realmente influenciam investimentos, ao afetarem a estrutura e a intensidade competitiva nos mercados de plataformas de redes. Ao se estabelecerem regras para transações entre detentoras de plataformas distintas e operadoras de redes voltadas à prestação de serviços, tais políticas tentam estabelecer um ambiente competitivo equilibrado por meio da padronização de tais transações entre os atores de mercado, visando uma redução geral dos custos de transação. Nesse sentido, os instrumentos de regulação da infraestrutura de telecomunicações tendem a se encaixar em duas grandes categorias: regulação horizontal e regulação vertical.

Ao se realizar uma análise comparativa da eficiência das medidas adotadas em regulação horizontal, tais como direitos de passagem, compartilhamento, co-localização, interconexão, desagregação e revenda, dentre outros, e em regulação vertical, incluindo separação contábil, separação funcional, separação estrutural, assim como regras de nãodiscriminação, tais como, por exemplo, a neutralidade de rede, verifica-se que o saldo é mais favorável às medidas de regulação horizontal.

As medidas de regulação horizontal estão muito bem consolidadas e dispõem de amplo suporte conceitual e teórico. Não obstante, raramente há uma única medida ideal a ser implementada. Ao contrário, o uso de um conjunto de medidas regulatórias permite uma melhor adaptação a contextos específicos, além de permitir que se atinja um equilíbrio entre aspectos estáticos de eficiência no curto prazo, e aqueles de eficiência dinâmica no longo prazo. Um elemento-chave nessa estratégia é que estejam disponíveis instrumentos regulatórios que assegurem acesso aos meios de backhaul e de interconexão com backbones, de forma a que se consiga extrair o máximo benefício advindo da modularidade das tecnologias avançadas de redes de telecomunicações.

${ }^{10}$ BAUER, J. (2010). Regulation, Public Policy, and Investment in Communications Infrastructure. Telecommunications Policy 34: 65-79. 
No entanto, é importante observar que as operadoras de rede verticalmente integradas têm incentivos concretos para discriminar, e mesmo excluir, os competidores que não são verticalmente integrados. Aqui a questão é se a separação é a medida regulatória correta e, caso afirmativo, qual das modalidades de separação é a melhor forma de se lidar com o problema. Com relação às regras de não-discriminação, a percepção é de que a discussão ainda está em estágio inicial, embora alguns resultados preliminares indiquem que tais regras trazem benefícios para o desempenho setorial.

\section{Situação no Brasil}

Um dos objetivos expressos do Plano Nacional de Banda Larga do Brasil é o de aumentar a penetração dos serviços de banda larga, com a participação ativa de pequenas e médias empresas operadoras de redes e provedores de serviço de internet. Para esses atores de mercado, um dos aspectos que mais pressiona seu modelo de negócio atualmente é o custo de trânsito para escoamento de tráfego para a internet e a falta de meios para troca direta de parte deste tráfego, por meio de peering, localmente.

Diante da tendência atual de migração para o ambiente NGN, e na ausência de regulação setorial específica, as operadoras dominantes verticalmente integradas têm incentivos para lançarem mão de práticas discriminatórias em relação às operadoras que não são verticalmente integradas. Elas tenderão a estabelecer um reduzido número de pontos de interconexão IP, e não terão interesse em se fazer presentes em pontos neutros de interconexão. Nesse cenário, as operadoras dominantes detentoras de backbones não permitirão que as pequenas operadoras provedoras de acesso estejam visíveis no nível de roteamento IP das suas redes de transporte.

Dadas essas restrições, uma medida eficaz de regulação horizontal na área de interconexão IP seria um aumento no número e na distribuição geográfica dos PTT, de forma a oferecer a pequenas e médias operadoras de rede e prestadores de serviço as vantagens competitivas que representam a compra de capacidade de trânsito na internet e a troca de tráfego, por meio de peering, localmente. 
Além dos benefícios econômicos do estímulo à competição no mercado de atacado, no transporte IP, e de se assegurar um ambiente de condições mais isonômicas para os atores de mercado, a implantação dos PTT agrega as externalidades positivas da melhoria de topologia e da eficiência de roteamento, maior conectividade e redução da latência de rede. Dado que a utilidade de um PTT é diretamente proporcional ao número de redes presentes naquele ponto de troca, isso claramente parece justificar um requisito regulatório de pontos de interconexão abertos e neutros. Ademais, é essencial que as operadoras dominantes detentoras de backbones estejam dentre aquelas presentes nesses PTT.

Tal como já apontado em estudos sobre o tema ${ }^{11}$, as operadoras de backbones de internet se defrontam com incentivos conflitantes: de um lado há incentivos para cooperarem entre si, de forma a oferecer aos seus clientes acesso à mais ampla gama de conteúdo de internet possível e, de outro, há incentivos para competirem entre si, de forma a conquistarem o maior número possível de clientes de varejo e de atacado, para dominar o mercado.

A regulação no mercado de interconexão IP, e em especial a implantação obrigatória de PTT neutros, é ainda mais importante no Brasil, dado que todos os backbones de internet em fibra ótica tendem a convergir em um número muito reduzido de locais - sobretudo Rio de Janeiro, São Paulo e Fortaleza - onde se interconectam com os cabos submarinos, que provêem capacidade de transporte internacional para a internet. $\mathrm{O}$ acesso a esse recurso de gargalo é um dos fatores-chave na estrutura de preços do mercado de atacado de trânsito IP no país. Devido à posição de PMS das operadoras dominantes detentoras de backbones, é essencial que operadoras de redes menores também possam acessar essas instalações de interconexão, seja diretamente nesses locais, ou indiretamente por meio de uma malha nacionalmente distribuída de PTT interconectados.

No entanto, para usufruir dos benefícios de participar de um ambiente de condições mais isonômicas no mercado IP, um grande número de Provedores de Serviço de Internet (PSI) e de operadoras de redes de acesso ainda precisa evoluir para a categoria de Sistema Autônomo (AS). Felizmente esse é um passo relativamente simples de ser dado, uma vez que

${ }^{11}$ KENDE, M. (2000). The Digital Handshake: Connecting Internet Backbones. In: FCC OPP Working Papers 32. 
a regulação assegure, como benefício tangível, a oferta de pontos de troca neutros e abertos.

Como já mencionado em um importante estudo que aborda a complexidade das interconexões na internet, ${ }^{12}$ a era dos acordos de peering sem custo, e de trânsito com pagamento integral, foi adequada às regras de mercado de um período da internet que está se encerrando. No entanto, o ambiente de interconexão está se tornando cada vez mais complexo, e o surgimento de novos tipos de contrato gera eficiências intrínsecas e autoaplicáveis, disponíveis a todos os sistemas autônomos.

Nesse novo ambiente, conforme relata recente artigo, ${ }^{13}$ os provedores de serviços e operadoras de rede que não possuem tráfego suficiente para realizarem peering com as operadoras de backbones constatam, no entanto, que possuem volume de tráfego suficiente para realizarem peering com outras redes regionais, no que se convencionou chamar de peering secundário. Essa troca de tráfego reduz os custos para os usuários finais e aumenta o controle sobre a qualidade de serviço. O peering secundário reduz o volume de trânsito que precisa ser adquirido por redes regionais, e permite que sejam acessados diretamente os clientes atendidos por seus demais parceiros de peering secundário. Assim, essas redes passam a adquirir trânsito parcial, que cobre somente parte de seu volume total de tráfego na internet.

\section{Conclusões}

Os argumentos em favor de uma regulação mais ativa no mercado de interconexão IP parecem ter amplo apoio, como indicam estudos recentes na literatura especializada. Como, atualmente, vários países discutem seus planos nacionais de banda larga, que, em alguns casos, incluem a estruturação de novos backbones nacionais de fibra ótica, é importante ressaltar que tais planos deveriam também abranger medidas regulatórias

\footnotetext{
${ }^{12}$ FARATIN, P. [et al.]. (2007). Complexity of Internet Interconnections: Technology, Incentives and Implications for Policy. In: The $35^{\text {th }}$ Annual Telecommunications Policy Research Conference.

${ }^{13}$ YOO, C. (2010). Innovations in the Internet's Architecture that Challenge the Status Quo. In: Journal on Telecommunications and High Technology Law 8: 7999. 
que assegurem acesso a essa infraestrutura de backbone e backhaul para as operadoras de rede e demais prestadoras de serviço de pequeno e médio porte. Nesse sentido, uma das ferramentas mais eficazes seria a implantação de uma ampla malha de PTT, que teria o efeito de melhorar a topologia de redes no nível de backbone e de prover um ambiente mais aberto para interconexão de redes menores com os backbones nacionais. Adicionalmente, medidas regulatórias específicas poderiam determinar a presença mandatória nesses PTT neutros por parte de todas as operadoras de backbones com posição de PMS.

\section{Bibliografia}

ATLAS. (2002). Connecting Scotland: our broadband future - Making it Happen. Scottish Executive.

BAUER, J. (2010). Regulation, Public Policy, and Investment in Communications Infrastructure. In: Telecommunications Policy 34: 6579.

BERKMAN CENTER FOR INTERNET \& SOCIETY. (2009). Next Generation Connectivity: A Review of Broadband Internet Transitions and Policy from Around the World. Harvard University.

CAMBINI, C.; JIANG, Y. (2009). Broadband Investment and Regulation: A Literature Review. In: Telecommunications Policy 33: 559-574.

CISCO. The Keystone of Internet Economy, Next Generation Interconnection. In: Cisco Networkers (2001-2005).

ERG. (2007). Project Team on IP-Interconnection and NGN: Final Report. European Regulators Group.

EURO-IX. (2009). Report on European IXPs - 2009. European Internet Exchange Association.

FARATIN, P. [et al.]. (2007). Complexity of Internet Interconnections: Technology, Incentives and Implications for Policy. In: The 35th Annual Telecommunications Policy Research Conference.

GETSCHKO, D.; MOREIRAS, A. (2008). Os Pontos de Troca de Tráfego, o PTT Metro e a internet brasileira. In: poliTICS 1: 15-21.

KENDE, M. (2000). The Digital Handshake: Connecting Internet Backbones. In: FCC OPP Working Papers 32. 
MARCUS, J.; ELIXMANN, D. (2008). The Future of IP Interconnection: Technical, Economic, and Public Policy Aspects. WIK Consult, European Commission.

YOO, C. (2010). Innovations in the Internet's Architecture that Challenge the Status Quo. In: Journal on Telecommunications and High Technology Law 8: 79-99. 